\title{
RिUCES
}

Revista Nicaragüense de Antropología

\section{EXPERIENCIAS ETNOGRÁFICAS}

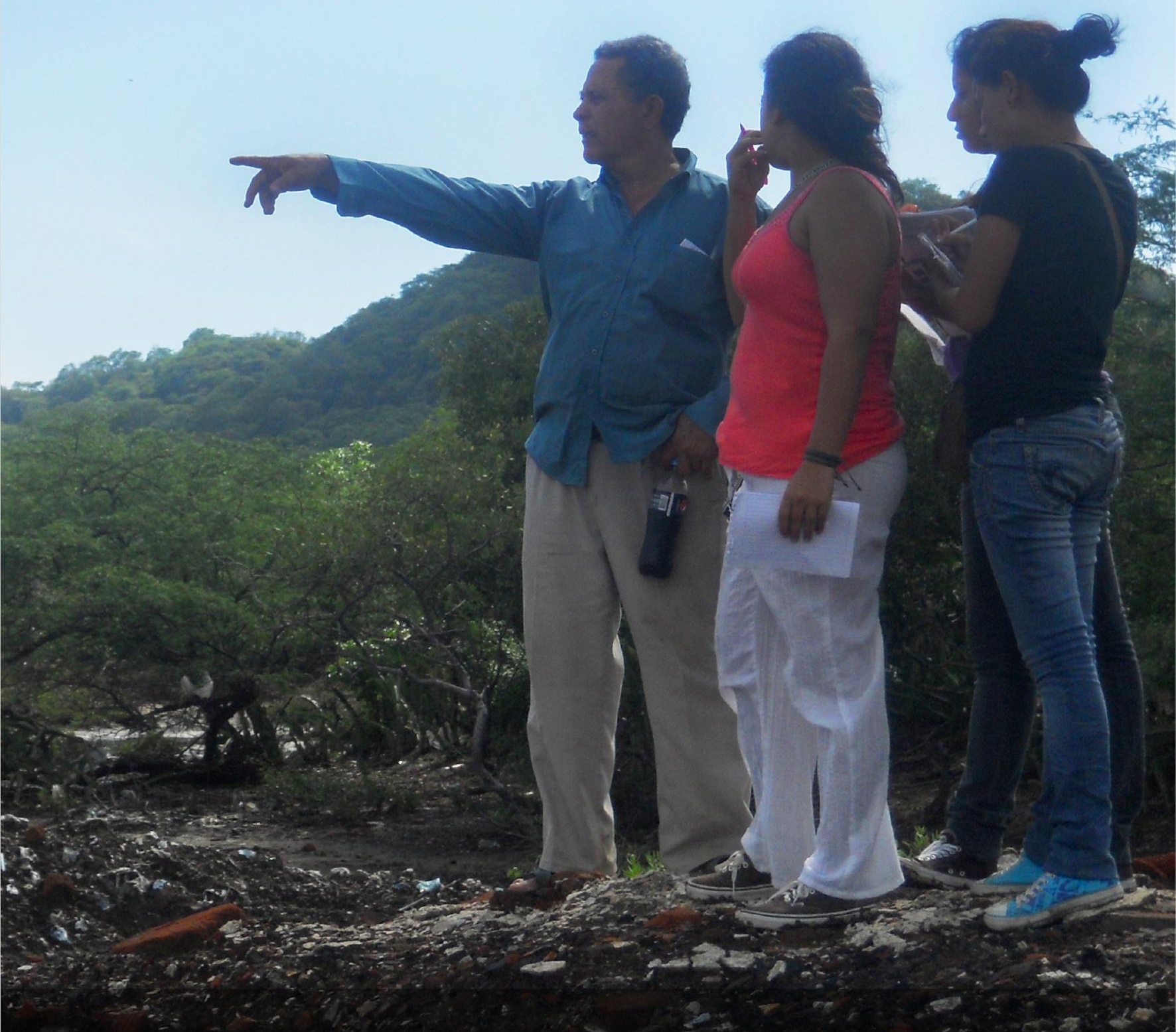

Quien investiga recurre a los conocimientos de la población para que le muestre el camino a seguir en la búsqueda de la compresión profunda de la cultura local. El o la investigadora también acompaña a la comunidad en su proceso de re-descubrirse, re-pensarse y proponerse. En la fotografía, un poblador les muestra a estudiantes de Antropología las características del territorio y la relación de éste con la población y su vida cotidiana en Salinas de Nahualapa, Tola, Rivas.

(Fotografía: Xavier Rodríguez) 


\title{
Hacia un relato polifónico: experiencias de campo en la Ciudad de La Plata
}

\begin{tabular}{|c|c|c|}
\hline Griselda Späth & Mora Ruffo & Araí ltatí Acuña \\
\hline $\begin{array}{l}\text { Orcid 0000-0002-9386-4419 } \\
\text { griseldaspath@gmail.com }\end{array}$ & $\begin{array}{l}\text { Orcid 0000-0002-5465-4996 } \\
\text { mora.ruffo@gmail.com }\end{array}$ & $\begin{array}{l}\text { Orcid 0000-0001-7164-9702 } \\
\text { araia8@hotmail.com }\end{array}$ \\
\hline Rosana Menna & Julián Trovero & Gonzalo Schmidt \\
\hline $\begin{array}{l}\text { Orcid 0000-0002-3424-2257 } \\
\text { rosana.menna@gmail.com }\end{array}$ & $\begin{array}{l}\text { Orcid 0000-0001-6735-299X } \\
\text { juliantrovero@gmail.com }\end{array}$ & $\begin{array}{l}\text { Orcid 000-0002-4177-3988 } \\
\text { schmidt_gonzalo@yahoo.com.ar }\end{array}$ \\
\hline
\end{tabular}

\section{Resumen}

Si como sostiene Herzfeld (1987) "la antropología, es la práctica de una teoría", la práctica de la disciplina se aprende en el "Trabajo de Campo". Aprender a distinguir, a observar, a escuchar, a saber interrumpir y a registrar. Partiendo de una práctica de entrenamiento en trabajo de campo en la ciudad de La Plata, Argentina, pretendemos en este artículo debatir sobre la construcción de un conocimiento colaborativo, intentando derribar esos mitos arraigados del genio del/la antropólogo/a en soledad, aislamiento y exotismo. Desde una narrativa polifónica, acorde al desarrollo de la investigación, compartimos algunas reflexiones sobre este rito de iniciación en el oficio del antropólogo y los avatares de la escritura. Poniendo de manifiesto la tensión, entre lo individual y lo grupal, que entendemos constituyen en su dialógica, el meollo de la construcción del conocimiento colaborativo.

Palabras clave: Experiencia, Trabajo de campo, Antropología Urbana, Conocimiento colaborativo, Método

\begin{abstract}
If, as Herzfeld (1987) sustains "anthropology is the practice of a theory", the practice of the discipline is learned on the "fieldwork". To learn to distinguish, to watch, to listen, to know when to interrupt, and to register. In this article we discuss the construction of a collaborative knowledge trying to tear down those ingrained myths of the anthropologist / genius in solitude, isolation and exoticism, starting from a training practice on field work in the city of La Plata, Argentina. From a polyphonic narrative, according to the development of the investigation, we share some reflections on this initiation rite in the profession of the anthropologist and the avatars of writing. Highlighting the tension between the individual and the group, what we understand constitutes, in its dialogic, the core of the construction of collaborative knowledge.
\end{abstract}

Key words: Experience, Fieldwork, Urban Anthropology, Collaborative Knowledge, Method. 


\section{Introducción}

\section{Hacia Un Relato Coral}

El espacio urbano enfrenta a los antropólogos, y nos ha enfrentado en cuanto aprendices del oficio (Augé, 2007; Bourdieu et al., 1999), a requerimientos particulares en el trabajo de campo, como la necesidad de llevar a la práctica ese mecanismo de desdoblamiento tan personal y siempre requerido: "el extrañamiento" (Augé, 1996; Althabe, 1999).

Hacer antropología en un presente próximo sugiere una serie de condiciones para producir conocimiento desde el interior del mundo social a escala microscópica. Los sujetos agrupados en la "situación de campo" pertenecemos a una pluralidad de situaciones sociales. Desde donde estemos, sólo obtendremos una presentación de sucesos, relaciones e intercambios bajo la forma de una "puesta en escena", que surge en el encuentro con los sujetos, y cuyo sentido debe primar en la misma situación:

El investigador es uno de los actores del juego social que se ha dado a la tarea de comprender, cuya coherencia quiere poner en evidencia, cuyas reglas quiere definir, permite tratar a la investigación misma como un terreno de indagación. La manera en que el antropólogo queda 'convertido' en actor, las transformaciones de su posición, las relaciones en las cuales se ve implicado, forman parte del universo social que estudia y son construidas por el modo de comunicación que elabora los términos que en él se emplean" (Althabe,1999, p.65).

La investigación en la que trabajamos comenzó cuando movidos por el interés de experimentar algo vinculado al oficio antropológico, Rosana, profesora de una de las materias que habíamos cursado recientemente, nos sugirió hacer "algo".

El objeto de estudio, los objetivos, y la metodología no los teníamos demasiado claros, empezamos con la idea de estudiar el espacio público. Estábamos casi todos por terminar el segundo año de la carrera de Antropología, y la estrategia de Rosana para transmitirnos el modus operandi del quehacer antropológico fue guiarnos con pocas instrucciones, algunas correcciones y muchos ejemplos al estilo de los manuales de metodología. Como señalan Bourdieu y Wacquant (1995), de una forma más parecida al entrenador deportivo que a un profesor.

Como actores del juego social que tratábamos de comprender nos proponemos hoy, jugar con la memoria y reflexionar sobre la práctica de entrenamiento en el trabajo de campo en nuestra ciudad: La Plata.

\section{Desarrollo}

\section{Nuestro espacio urbano}

La ciudad de La Plata - capital de la provincia de Buenos Aires, Argentina - fue fundada el 19 de noviembre de 1882. La idea rectora de Dardo Rocha, el gobernador de Buenos Aires, era "fundar una gran ciudad cuyo trazado y características urbanas sintetizasen condiciones de: higiene, las conquistas del arte y adelantos de la industria" (De Paula, 1987, p.77).

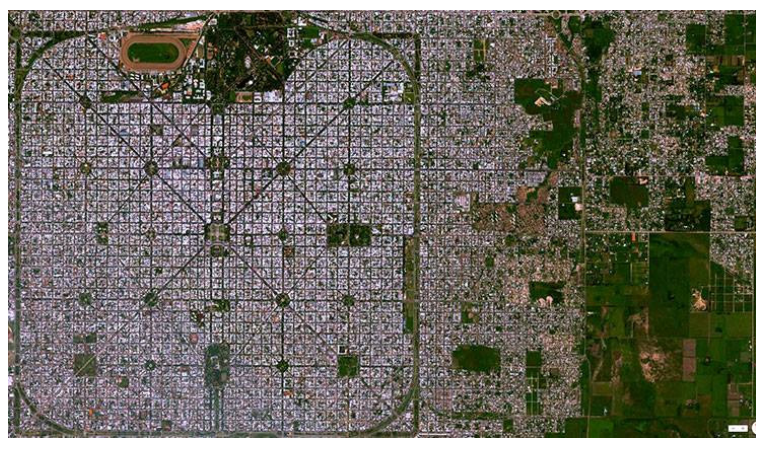

Vista aérea de la ciudad de La Plata, donde se aprecia el trazado fundacional. Fuente: Pousta

Así el trazado de la misma resultó clave para llevar adelante el proyecto urbano que se propuso. En palabras de este autor: "La ciudad de La Plata se materializó como un reflejo del urbanismo de fines del siglo XIX en la amplitud de sus calles y avenidas arboladas que aseguraban comodidad, ventilación e higiene; también se hizo presente aquel espíritu en la abundancia de sus plazas, parques y su Paseo del Bosque, providencialmente salvado por el Dr. Rocha" (De Paula, 1987 p.88). Dentro de esta lógica en 1883 se creó una comisión cuyo fin era encargarse del arreglo de las plazas públicas. Plaza Moreno, siendo el centro geométrico de la ciudad, reúne los edificios de importancia cívica local: la Catedral y la Municipalidad. Con algunas excepciones, la mayoría de las plazas se configuran como rotondas, cuadrados en 
posición ortogonal o diagonal, lo que genera distintos diseños de la trama urbana.

Al día de hoy, como pudimos apreciar durante nuestro trabajo de campo, las plazas permanecen como espacios públicos, manteniendo el espíritu original del trazado y la relevancia en la vida cotidiana de sus habitantes.

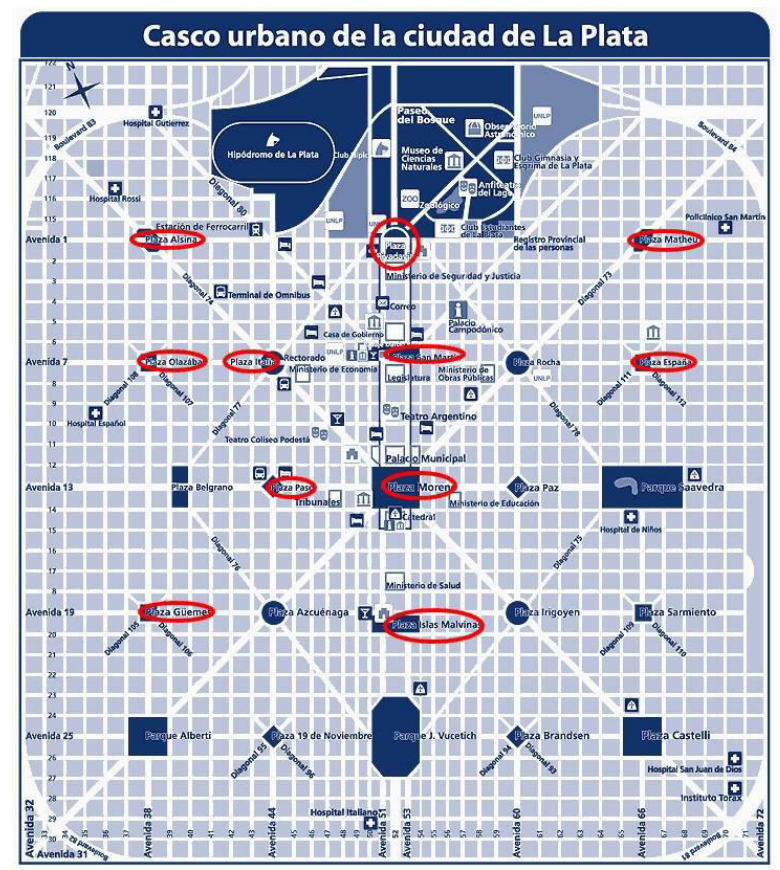

Plano de la Ciudad de La Plata con las plazas donde se realizaron las observaciones y entrevistas, marcadas en rojo. La Plata, 2014 Fuente: Web Gifex

La investigación fue un proceso largo, que abarcó varios momentos y formas. Fuimos construyendo y delimitando el objeto a medida que avanzábamos en el trabajo de campo y en las lecturas. Algunos de nosotros comenzamos leyendo y en un momento posterior salimos al campo, mientras que otros a partir de las inquietudes allí surgidas, pasamos a la reflexión teórica, mediada nuevamente por la lectura.

Con la idea de refractar nuestras diferencias en tanto actores sociales plurales de la misma investigación, abrimos este trabajo con una presentación polifónica de nuestras experiencias.

\section{Contrapunto de Solistas:}

\section{Mora}

Mi primera participación en el grupo fue en plaza Moreno. Tras faltar a la reunión previa, llegué al campo con un: "Llévate una libreta y llega a las dos, nos encontramos en el medio de la plaza". Decir perdida, es poco. Ese primer día copié las preguntas que habían hecho y acompañé a los demás en sus entrevistas. Me dediqué a observarlos a ellos y a hacer, muy de vez en cuando, comentarios. Durante las primeras reuniones recuerdo que no participé mucho, me centré en mirar la dinámica del grupo y ver qué era lo que Ros nos presentaba y nos pedía, no me sentía lista para participar del todo y preferí empezar en silencio. Como yo no soy de la ciudad, ir a las plazas fue conocer nuevos lugares y ampliar un poco el espacio por el que me manejaba. Desde un principio me emocionó mucho el juntarnos a "hacer antropología", lo que fuera que eso quisiera decir.

\section{Julián}

Yo ingresé a la facultad en el año 2009. El entusiasmo me duró poco. Al año decidí regresar a mi casa, en los alrededores de la ciudad, y dejar mi residencia en La Plata. En ese momento tuve una crisis con la carrera. La delegué al último plano. Pero casi como una obligación no perdí la constancia, sobre todo, con los exámenes.

A las pocas semanas, Rosana nos ofreció, a algunos compañeros y a mí, ingresar al laboratorio de la División Etnográfica. La propuesta era trabajar sobre las plazas de nuestra ciudad; algo así como "el uso del espacio público". Nunca fue muy clara. Acepté. Casi sin querer. No me sentía cómodo en ninguna clase, en ningún laboratorio, sin embargo, acepté.

Las plazas fueron una buena experiencia. Me acuerdo del miedo que tenía antes de ir a la primera. Llevábamos algunas preguntas para hacer entrevistas. Incertidumbre era todo lo que teníamos. No me sentía en condiciones de trabajar. ¿Cuándo cambié mi actitud? Creo que fue cuando una chica más o menos de mi edad se sentó a descansar en un banco. La entrevisté, algo me gustó en ella, me dejé llevar por la situación. Sin importar la información que saqué o que perdí esa fue mi primera entrevista, o por lo menos la primera de la que tengo buen recuerdo. Desgrabar fue una experiencia horrenda. Había que desgrabar por alguna razón. 
Es lo que hacen los antropólogos. Tampoco me sentía antropólogo.

\section{Araí}

¿Cómo empecé mi trabajo? venía de terminar un primer año muy difícil relacionado mayoritariamente a las ciencias naturales. En segundo año comenzó eso que había anhelado y con ello el descubrimiento de la carrera, materia tras materia. Por esa época, una profesora nos invitó a hacer trabajo de campo en las plazas, ¿En las plazas? Lugar que desde siempre me trajo buenos recuerdos, cargados de vivencias gratas y hermosas experiencias, ¿Cómo decir que no? Me entregué sin duda. Entrevistas, observaciones, grabaciones, palabras que escuchaba decir a diario, pero no comprendía aún su significado... Con el tiempo, pude entender que esta primer experiencia fue muy importante en el desarrollo posterior de todos mis trabajos de campo, mis entrevistas, hasta incluso el lugar donde elegí trabajar (el proyecto de investigación) ya que al haberlo hecho de una manera colaborativa, y en grupo, posteriormente siempre busqué experiencias similares; no en la soledad que requieren ciertos trabajos antropológicos. Considero que nuestra primera entrada al campo modela la práctica profesional, inscribiéndose en el cuerpo.

\section{Gonzalo}

Todo comenzó con una inquietud, un interés que se remitía a la cuestión de tener una experiencia en el aprendizaje acerca del quehacer antropológico. Se asomaba una imagen idílica producto de lecturas de aquellos autores clásicos que nos relataban acerca de tierras lejanas en sus etnografías. No obstante, ante el peso de la evidencia del momento histórico reciente y geográficamente cercano, se convertía aquello en una representación casi novelesca. Narrativas insoslayables, por la riqueza de sus datos y contribuciones para pensar el trabajo de campo, sin duda aportaron al ejercicio de la antropología para estar ahí. En este caso, para estar en una plaza observando "un detalle que yo reconozca significativo según mis ideas de lo que constituye una diferencia", lo cual era un reto y una apuesta por la antropología urbana como persona que habita esta misma ciudad: situarse en el campo y no sólo en una plaza de mi cotidianidad.

\section{Rosana}

A pesar del tiempo transcurrido, siempre recuerdo a "mis" estudiantes, y sí, digo bien "recuerdo", dado que esta palabra con un origen tan latino: "ricordo"; y como me han dicho los etimólogos, sería su significado último y escondido "volver a pasar por el corazón". Así nace la propuesta de trabajar "juntos" para que pudiéramos narrar, lo que fue para ellos el ineludible "rito de pasaje": el trabajo de campo.

Casi todos los años, al terminar la cursada, se acercan los/ las estudiantes para conocer la "cocina" de los trabajos de investigación. La mayoría de las veces acobardados/as por la tarea inmensa, hercúlea que suponen deben realizar: escribir una obra como la de los grandes clásicos que han leído dentro de la currícula, intrigados por trasponer la teoría en trabajos específicos. Así, se fueron formando los grupos con aquellos motivados por conocer. $Y$ en esos vínculos, fueron descubriendo fortalezas y debilidades, que posibilitan ir asumiendo roles, compromisos, gustos e ideologías; aspiraciones y sueños.

Desde una formación y experiencia metodológica de índole cualitativo descriptivo, suelo estar muy atenta a la sinderepia (Pardinas, 1993), pues en este tipo de abordajes es de mucha importancia tener en cuenta quienes la realizan, según sean las motivaciones de cada recorrido biográfico. Por ello planteo temas o topos muy amplios, así afloran esas preguntas minúsculas, pero que un trabajo reflexivo puede convertirlas en un "objeto" de investigación. Mayormente, la experiencia y alguna lectura me ha indicado que una cuota de misterio, seduce (Baudrillard, 1981), despierta la pasión y aflora la creatividad.

\section{Un trabajo en conjunto}

Nuestra ida al campo fue un proceso de reflexión continua, circunscribimos en simultáneo las técnicas de construcción de datos, y los conceptos que aplicábamos. Realizamos observaciones que registramos en libretas de campo, tanto en forma gráfica cómo narrada, grabamos entrevistas personales semi-estructuradas, individuales y grupales (focus group naturales, Marradi et al., 2007) y tomamos fotografías digitales.

Revisábamos en el laboratorio las entrevistas para ver cómo podíamos mejorar nuestra performance en el campo, empezamos apegándonos a las preguntas que 
habíamos diseñado y a medida que nos sentimos más cómodos las flexibilizamos. También fuimos ajustando el diseño de las mismas, para asegurarnos que las respuestas no fueran influenciadas. En el campo aprendimos a "escuchar", a poner en práctica nuestras incipientes reflexiones metodológicas.

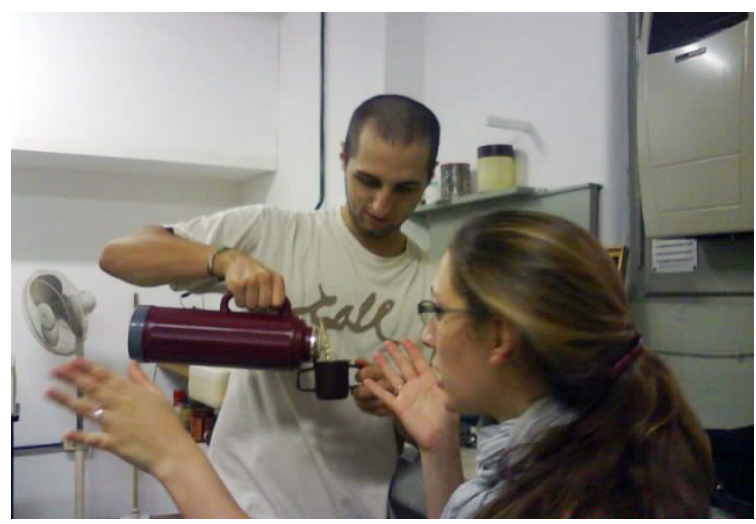

En el laboratorio de la División Etnografía del Museo de La Plata FCNyM - UNLP, siempre se compartían discusiones y mate (bebida autóctona Argentina). La Plata, 2011(Autora: Rosana Menna)

Del ejercicio de observación entendimos que teníamos que delimitar un contexto. Entonces nos propusimos aunar criterios en la forma de registrar, ya que los datos que cada uno había construido no eran homologables. $\mathrm{Al}$ inicio, cada uno tenía diferentes intereses, la mayoría de nosotros comenzó sin ninguna lectura, pretendiendo que el campo nos abriera los interrogantes. Despertado el interés, buscamos en la lectura las herramientas para enfrentar nuevamente la tarea e ir completando (en el campo) el ejercicio de cada una de las técnicas. Así, casi sin notarlo, fuimos armando nuestra caja de herramientas repleta de experiencias, técnicas y teoría, indispensable para el oficio del antropólogo.

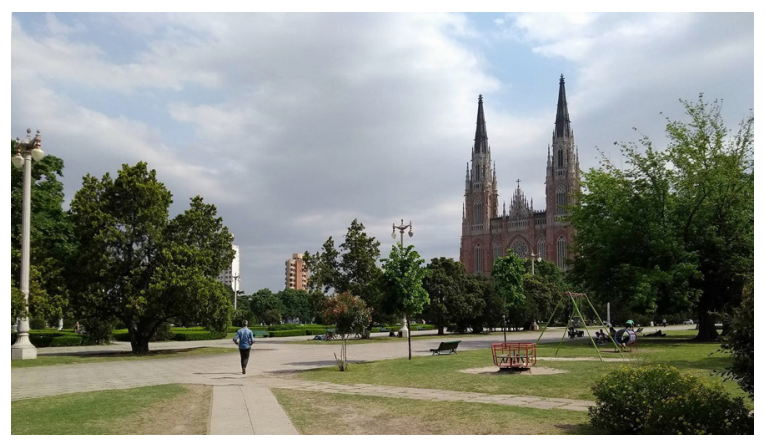

El hecho de que el trabajo se realizara en nuestra ciudad nos obligó a repensar el entorno, delimitándolo como objeto de estudio. Vivir en la ciudad o en sus alrededores hizo que ir al campo pareciera sencillo, en el sentido de que estaba cerca, siempre ahí, a nuestro alcance.

La práctica nos mostró que "estar ahí" como antropólogo implicaba un cambio de actitud momentáneo nada fácil de realizar: no fue algo intensivo, no nos instalamos un largo período de tiempo en un contexto completamente diferente al de nuestra cotidianeidad. En un principio el "extrañamiento" fue un esfuerzo consciente; un desafío que con el tiempo fuimos internalizando. Nunca lo logramos del todo. Conocíamos los lugares de distintas maneras: los que vivimos toda la vida en la ciudad teníamos una perspectiva diferente de aquellos que recién llegábamos; todas las miradas aportaron a la construcción de la perspectiva antropológica.

\section{La Partitura}

\section{Rosana}

Esta narrativa, cómo el trabajo mismo, se construyó desde el estilo y la creatividad de todos los que participamos. Fue mi interés promoverlo como facilitadora de conocimiento, pues no me sentiré nunca una docente que tiene "el saber". Por ello, en un intento un tanto atrevido de emular la "pluma" de Oscar Lewis en "Una muerte en la familia Sánchez", buscamos dejar plasmadas las vivencias de tan significativo ritual para la vida profesional. Tratando al narrar de incorporar la performance experiencial en semiosis escritural. El primer trabajo de campo, es de esas vivencias que quedan tatuadas en la memoria. No es el primer grupo, que después de cursar la materia Teoría Antropológica, queda seducido por el reflexionar sobre el trabajo antropológico, anhelando participar de ese ir y venir; entre la más abstracta teoría y el más misterioso y palpable campo; entre la lectura, la acción y la reflexión sobre lo actuado. Todos/as hemos debido pasar por este ritual. Con otros grupos hemos trabajado en nuestra ciudad, La Plata, en los edificios públicos, en los bares y en los tiempos de "la fiesta". Todos estos trabajos fueron motivos de encuentros con estudiantes curiosos/as y apasionados/as que forjaron lazos que se mantienen a pesar del tiempo y la distancia.

Plaza Moreno, centro geográfico de la ciudad. La plata, 2017 (Autora: Mora Ruffo) 
Y lo más entrañable ha sido que ha surgido el afecto, por ese trabajar solidariamente, colaborativamente y parafraseando a Sennett (2012): cuando más y mejor se coopera, la habilidad social y la cognitiva se entremezclan. Las dos habilidades dignas de destacar son: el experimento y la comunicación. Experimentar implica hacer cosas nuevas, y más aún, estructurar estos cambios en el tiempo. La comunicación, casi siempre ambigua, con el tiempo requiere pactar las reglas de juego, ser capaces de negociar y resolver. Se advertirá que aprender a cooperar en estos términos no es nada fácil. Esta dificultad, en cierto sentido, es positiva; la cooperación se convierte más en una experiencia adquirida que en un mero compartir irreflexivo. Lo mismo que en otros campos de la vida, apreciamos más lo que hemos conseguido con esfuerzo.

\section{Griselda}

También yo llegué aquí, convocada por Rosana, con quien en su momento me inicié en los avatares del trabajo de campo antropológico... y desde entonces, con más o menos intensidad, seguimos trabajando juntas... Como eslabón en esta cadena de formación que emprende cada año, estar incluida en otro lugar del camino de investigación/narración, me enfrentó a un desafío diferente: ya no el del momento fundante del trabajo de campo, sino el de la construcción de una narración colaborativa.

¿No es con otros, cómo llevamos a cabo cada paso del proceso de investigación? Y en esta oportunidad, también el de narración. Entonces cada paso no puede menos que ser un arte. Un arte distinto y novedoso seguro, según quien sea ese otro, pero sin lugar a dudas será un arte o ciencia de trabajar colaborativamente.

\section{Julián}

No quiero hacer una antropología vacía. Una antropología desde los libros está vacía. Tampoco quiero escribir una antropología insulsa. La antropología sin creatividad está muerta. Nuestra disciplina nace en el campo.

Necesita de la veracidad del campo, de las relaciones que allí se erigen y se derrumban. Pero ¿Qué es la antropología? ¿Por qué hacemos antropología? ¿Para quién hacemos antropología?

Se dice que la antropología está en los libros, en parte estoy de acuerdo. Es fundamental un compromiso del antropólogo como autor, con su firma. El texto es un canal donde el escritor puede volcar su subjetividad. Distinguir no sólo su experiencia en el campo como un "estar allí", sino las partes de sombras e incertidumbres que una investigación jamás llega a disipar. Poder transmitirlo es entender la palabra como objeto, atravesada por distintos significados cuando es leída; su sonoridad, longitud, su aspecto visual, es un sustento que poco a poco delínean una imagen, como el trazo de un pintor. Cada palabra tiene luminosidad propia y afinidades con la naturaleza de la que quiere dar cuenta. ¿Cuáles son los relatos etnográficos más populares? Aquellos que no se privan del poder de la palabra ambigua (o poética, o palabra-objeto), esta palabra, leída desde el campo es siempre esclarecedora, porque es sincera. Pero hacer antropología no es hacer poesía. La escritura está en el corazón de la disciplina. Pero escribir no es hacer antropología. La primera aproximación al campo me sembró una importante pregunta: ¿Qué antropología quiero hacer?

\section{Gonzalo}

La situación de campo en plazas de mi cotidianidad, puso en escena la reflexión sobre mis condicionamientos en tanto sujeto portador de la misma condición ciudadana que abordamos etnográficamente. Aquella "experiencia ritual" estaba empalmada también con la condición de ser un joven estudiante y extranjero. Atravesamientos que son considerados para hacer una antropología situada. En el transcurso de esta experiencia de antropología urbana, el extrañamiento formó parte del proceso. A mi entender, resulta productivo en lo metodológico, y a partir de esta experiencia, tomar decisiones sobre la condición de sujeto en el campo, para ejercer la situación de extrañamiento.

\section{Discusión}

La estrategia narrativa polifónica no es novedosa. Fue propuesta como una maniobra, en los surgimientos de la antropología posmoderna, para diseminar la autoridad del autor (Clifford y Marcus, 1991; Geertz 1989). Si bien este estilo de escritura fue insinuado para el par Antropólogo/Informante, este trabajo reformula el procedimiento en pos de enaltecer la pluralidad de voces autoriales. Pluralidad que intenta reflejar la diversidad de experiencias que integran el proceso de investigación. Éste estuvo planteado desde el inicio en clave grupal. Implicó negociaciones, revisiones 
y resoluciones que desembocaron en un quehacer colaborativo. Los segmentos individuales y grupales del texto buscan honrar la lógica de las experiencias en el campo y laboratorio; respetando la dialógica de la convivencia.

En el lapso de un año, relevamos diversas plazas de la ciudad, intercalando integrantes, días y horarios. Todos trabajamos eventualmente con cada uno de nuestros compañeros, volviendo imposible de separar el trabajo singular del trabajo del equipo. Fuera del campo, las lecturas, si bien individuales, eran compartidas en las reuniones, discutidas y aplicadas a nuevas instancias de investigación.

El antropólogo también es un ser social. Con los otros en el campo, y con los otros en la academia. Las trayectorias individuales pertenecen a una misma urdimbre. Esa ambigüedad, esa tensión, entre el actor y el escenario, entre el músico y la orquesta, constituyen el fundamento de este artículo.

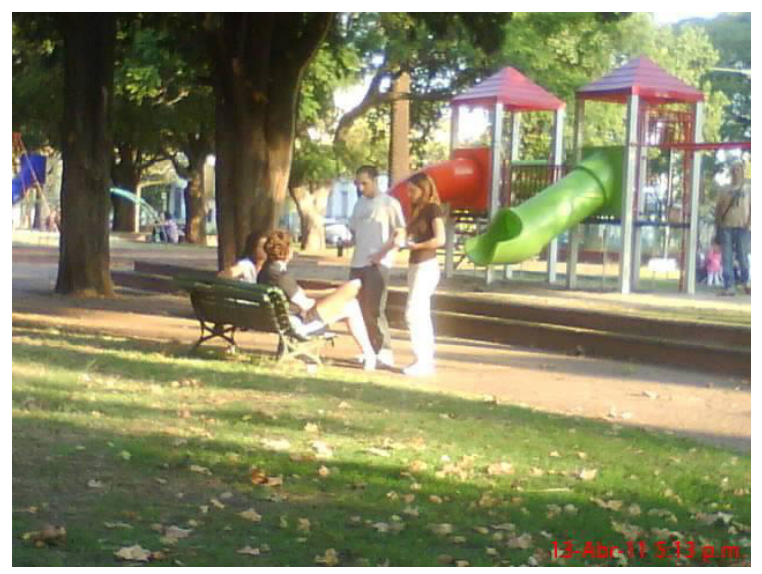

Realizando una entrevista en Plaza Alsina. La Plata, 2011 (Autora: Rosana Menna)

\section{Conclusiones}

La redacción de este trabajo indica que se consumó un recorrido. Nuestras reflexiones surgieron a partir de la práctica y de las lecturas, así como de los intercambios, y discusiones.

Pero toda esa actividad del orden del deseo y la intención, permanece anárquica o se vuelve efímera si no es plasmada en la escritura. El pasaje de una forma oral a una escrita no implica sólo un ejercicio de estilo, sino una elaboración conceptual.

La forma en que nos iniciamos en el trabajo de campo nos permitió considerar al proceso de investigación inserto en una red de relaciones que abarcan más de lo que las grandes etnografías dejan entrever. El antropólogo solitario no existe, ni siquiera en la más espesa de las selvas. Trabajar en equipo es un desafío, la producción colaborativa implica concesiones y pérdidas, pero también permite emergentes inalcanzables por otros caminos. La ciencia nos trae una encrucijada: años de hiper-especialización, individualización y competencia entre los científicos han renovado la crítica ¿Cómo queremos trabajar? Con otros y no contra otros. No sólo entre las distintas disciplinas, sino dentro de la misma. El diálogo y el debate constante, son quienes engrandecen esta manera peculiar. Porque en el espacio que tenemos en frente, en las costas que conforman lo ajeno, se esconde todo lo que no somos, todo lo que no sabemos, todo lo que queremos ser. En palabras de un poeta uruguayo:

\section{"[...] Así pues}

desde este misterioso confín de la existencia los otros me ampararon como árboles con nidos o sin nidos poco importa no me dieron envidia sino frutos [...]"

\section{(Como árboles, Mario Benedetti)}

\section{Bibliografía}

Althabe G. y Schuster F. G. (comp.), (1999) Antropología del presente, Buenos Aires. Argentina: Edicial

Augé, M. (1996) Los «no lugares» espacios del anonimato. Una antropología de la Sobremodernidad. Barcelona, España: Gedisa

Augé, M. (2007) El oficio del antropólogo. Barcelona, España: Gedisa

Baudrillard, J. (1981) Sobre la seducción. Madrid , España: Ediciones Cátedra, S. A. 
Bourdieu P. y Wacquant L. J. (1995) Respuestas: por una antropología reflexiva, México

Bourdieu, P. Passeron J.-C., Chamboredon J.C. (2002) El oficio de sociólogo, Buenos Aires, Argentina, Siglo XXI, (1999) México: Grijalbo

del Olmo, M. (2010) Dilemas éticos en antropología. Las entretelas del trabajo de campo etnográfico. Madrid, España: Ed. Trota

Clifford, James y Marcus, George E. (1991) Retóricas de la Antropología, Madrid, España: Ediciones Júcar

De Paula, A. (1987) La ciudad de La Plata: sus tierras y su arquitectura. Buenos Aires Argentina: Ediciones del Banco de la Provincia de Buenos Aires, Capital Federal

Geertz C. (1987) La interpretación de las culturas. México: Gedisa

Geertz C. (1989) El antropólogo como autor, Barcelona, España: Paidós

Hammersley M., Atkinson P. (2013) Etnografía, métodos de investigación. Barcelona, España: Paidós

Herzfeld, M. (1987) Anthropology through the LookingGlass: Critical Ethnography in the Margins of Europe. Cambridge, Great Britain: Cambridge University Press
Hornstein L. (2013) Las encrucijadas actuales del psicoanálisis. Subjetividad y vida cotidiana. Buenos Aires, Argentina: Fondo de Cultura Económica

Marradi, A, Archenti, N y Piovani, J.I. (2007) Metodología de las ciencias sociales. Buenos Aires. Argentina: Emecé Editores

Pardinas, F. (1993) Metodología y técnicas de investigación. Buenos Aires, Argentina : Siglo XXI

Sartre, J. P. (2008) ¿Qué es la literatura?, Buenos Aires, Argentina: Ed Losada SA

Taylor S. J. , Bogdan R. (1994) Introducción a los métodos cualitativos de investigación, Buenos AiresBarcelona-México: Paidós. (1987) Barcelona, España: Paidós

Verón, E. (1994) De l'image semiologique aux discursivités. Le temps d'une photo ["Discursividades de la imagen", La imagen fija. París, Centro Georges Pompidou, La Documentación francesa - From the semiotíc image to discursivity: the time of a photo] Hermès, Revue de l'Institut des sciences de la communication du CNRS (ISCC) - n 13-14 : Espace

Publics en Images- pp:45-64 http://documents.irevues. inist.fr/handle/2042/15084

\section{Griselda Späth}

Licenciada en Antropología sociocultural, por la Facultad de Ciencias Naturales y Museo de la Universidad Nacional de La Plata (FCNyM), también realizó estudios en Letras en la misma casa de estudios. Becada por el CONICET, inició su doctorado sobre construcciones identitarias vinculadas a las migraciones. La tesis doctoral, actualmente en evaluación, fue fruto de años de trabajo de campo, realizando entrevistas en profundidad a hijos de familias en tránsito.

Participa en distintos programas vinculados al ingreso y permanencia de estudiantes universitarios en la facultad y forma parte de un proyecto de extensión vinculado a la mejora de la calidad de atención en salud.

Actualmente trabaja como docente en el Departamento de Ciencias Sociales de la Facultad de Ciencias Médicas, en la cátedra de Epidemiología, y en la cátedra de Teoría Antropológica de la FCNyM. También se desempeña como docente, en el ámbito de la metodología de investigación, en la Universidad Maimónides. 


\section{Rosana B. Menna}

Licenciada en Antropología por la FCNyM - UNLP, también realizó estudios en Ciencias Jurídicas y en Docencia Universitaria en la misma Universidad. Trabaja desde hace 25 años en el Consejo Nacional de Investigaciones Científicas y Técnicas (CONICET), durante 21 años en el Programa PINACO dirigido por el Dr. Héctor B. Lahitte. Coordina desde 2002 el Proyecto Fenómenos Identitarios Urbanos en la División Etnografía del Museo de La Plata FCNyM - UNLP, dirigido por el Dr. Héctor B. Lahitte. Es docente de la Cátedra de Teoría Antropológica en la Facultad de Ciencias Naturales y Museo (FCNyM) de la Universidad Nacional de La Plata (UNLP); también es docente de Metodología en Ciencias Sociales, y de Antropología Social y Jurídica, estas últimas dos materias las dicta en la Universidad Nacional de Lanús (UNLa).

\section{Mora Ruffo}

Licenciada en Antropología, por la Facultad de Ciencias Naturales y Museo de la Universidad Nacional de La Plata (2016). Participó como pasante en el Proyecto Fenómenos Identitarios Urbanos dirigido por el Dr. Héctor B. Lahitte en la División Etnografía Facultad de Ciencias Naturales y Museo, UNLP (2010-2011 y 2013-2014).

Actualmente colabora con el Proyecto de investigación "Prácticas y discursos sobre- discapacidad, cuerpo y sociedad" y forma parte del proyecto de extensión "Jugándo-nos en la diversidad. Discapacidad e inclusión en una realidad en transformación” enfocado en la inclusión a través del juego.

\section{Julián Trovero}

Licenciado en Antropología sociocultural, por la Facultad de Ciencias Naturales y Museo de la Universidad Nacional de La Plata, FCNyM (2016). Estudiante de la maestría en Historia y Memoria ofrecida por la Facultad de Humanidades y Ciencias de la Educación (FaHCe) de la Universidad Nacional de la Plata.

Participó en el Proyecto Fenómenos Identitarios Urbanos dirigido por el Dr Héctor B. Lahitte División Etnografía Facultad de Ciencias Naturales y Museo, UNLP (2010-2011 y 2013-2014).

Actualmente trabaja en la continuación del proyecto "Historia Industrial de Villa Elisa desde los trabajadores", en el marco del programa Jóvenes y Memoria con los desaparecidos de la misma localidad en la dictadura cívico-militar del año 1976.

\section{Araí ltatí Acuña}

Licenciada en Antropología sociocultural, por la Facultad de Ciencias Naturales y Museo de la Universidad Nacional de La Plata (FCNyM). Becada por la Universidad Nacional de La Plata, inició su doctorado este año sobre construcciones identitarias vinculadas a niños y niñas con discapacidad. Se desempeña como docente adscripta en la facultad de Psicología desde el año 2015 a la actualidad.

Actualmente participa en el Proyecto de investigación "Prácticas y discursos sobre- discapacidad, cuerpo y sociedad" y forma parte del proyecto de extensión "Jugándo-nos en la diversidad. Discapacidad e inclusión en una realidad en transformación” radicados en la facultad de Humanidades y Ciencias de la Educación.

\section{Gonzalo Schmidt}

Licenciado en Antropología sociocultural,porlaFacultaddeCienciasNaturalesyMuseodelaUniversidadNacionaldeLaPlata,FCNyM. Participó en el Proyecto Fenómenos Identitarios Urbanos dirigido por el Dr. Héctor B. Lahitte, División Etnografía Facultad de Ciencias Naturales y Museo, UNLP (2010-2011 y 2013-2014). Actualmente participa del proyecto tetra anual "NOA. Arqueología, Historia y Paisaje" (11/N806, 2016 hasta diciembre de 2019) desarrollando investigación sobre los procesos de construcción social del patrimonio arqueológico en los sitios el Shincal de Quimivil, Catamarca, Argentina y Pucara de Chena en Santiago de Chile. 